\title{
A Modular, Reusable Latch and Decking System for Securing Payloads During Launch and Planetary Surface Transport
}

\author{
William R. Doggett ${ }^{1}$, John T. Dorsey ${ }^{2}$, Thomas C. Jones ${ }^{3}$ \\ NASA Langley Research Center, Hampton Va 23662 \\ Bruce D. King ${ }^{4}$ \\ Lockheed Martin, Hampton Va 23662 \\ and \\ Martin M. Mikulas ${ }^{5}$ \\ National Institute of Aerospace, Hampton Va 23662
}

Efficient handling of payloads destined for a planetary surface, such as the moon or mars, requires robust systems to secure the payloads during transport on the ground, inspace and on the planetary surface. In addition, mechanisms to release the payloads need to be reliable to ensure successful transfer from one vehicle to another. An efficient payload handling strategy must also consider the devices available to support payload handling. Cranes used for overhead lifting are common to all phases of payload handling on Earth. Similarly, both recent and past studies have demonstrated that devices with comparable functionality will be needed to support lunar outpost operations. A first generation test-bed of a new high performance device that provides the capabilities of both a crane and a robotic manipulator, the Lunar Surface Manipulation System (LSMS), has been designed, built and field tested and is available for use in evaluating a system to secure payloads to transportation vehicles.

A payload handling approach must address all phases of payload management including: ground transportation, launch, planetary transfer and installation in the final system. In addition, storage may be required during any phase of operations. Each of these phases requires the payload to be lifted and secured to a vehicle, transported, released and lifted in preparation for the next transportation or storage phase. A critical component of a successful payload handling approach is a latch and associated carrier system. The latch and carrier system should minimize requirements on the: payload, carrier support structure and payload handling devices as well as be able to accommodate a wide range of payload sizes. In addition, the latch should; be small and lightweight, support a method to apply preload, be reusable, integrate into a minimal set of hard-points and have manual interfaces to actuate the latch should a problem occur.

A latching system which meets these requirements has been designed and fabricated and will be described in detail. This latching system works in conjunction with a payload handling device such as the LSMS, and the LSMS has been used to test first generation latch and carrier hardware. All tests have been successful during the first phase of operational evaluations. Plans for future tests of first generation latch and carrier hardware with the LSMS are also described.

\footnotetext{
${ }^{1}$ Senior Research Engineer, Structural Concepts and Mechanics Branch, MS-190, Member, AIAA

2 Senior Research Engineer, Structural Concepts and Mechanics Branch, MS-190, Associate Fellow, AIAA

${ }^{3}$ Research Engineer, Structural Concepts and Mechanics Branch, MS-190, Member, AIAA

${ }^{4}$ Senior Designer, Structural Concepts and Mechanics Branch, MS-190

${ }^{5}$ Senior Research Fellow, Fellow AIAA
} 


\section{Nomenclature \& Abbreviations}

ELC $=$ Express Logistics Carrier

LSMS = Lunar Surface Manipulation System

ISS $\quad=$ International Space Station

\section{Introduction}

$\mathrm{E}$ fficient handling of payloads destined for a planetary surface, such as the moon or mars, requires robust systems to secure the payloads during transport on the ground, in-space and on the planetary surface. In addition, mechanisms to release the payloads need to be reliable to ensure successful transfer from one vehicle to another or to storage. Overhead equipment is widely used for payload handling in Earthbased operations. ${ }^{\text {i }}$ Similarly, both recent and past studies have demonstrated that devices with similar functionality will be needed to support planetary surface operations. $^{\text {ii, iii }}$ Typical payloads range from large habitation modules and lunar rovers to smaller prepackaged hardware and supplies, all of which must be unloaded from landers transported to their operational site and then accurately positioned there.

A payload handling approach must address all phases of payload management including: ground transportation, launch, planetary transfer and installation in the final system. In addition, storage may be required during any phase of operations. Each of these phases requires the payload to be lifted and secured to a vehicle, transported, released and lifted in preparation for the next transportation or storage phase. The payload handling approach must also be compatible with the available payload handling devices including forklifts and overhead cranes.

A critical component of a successful payload handling approach is a latch and associated carrier system. The latch and carrier system should minimize requirements on the: payload, carrier support structure and payload handling devices, while accommodating a wide range of payload sizes. In addition, the latch should be: small and lightweight, reusable, integrate into a minimal set of hard-points and have manual interfaces to actuate the latch should a problem occur. Ideally, the payload handling approach would reuse hard-points required for ground handling rather than requiring additional interfaces. Often launch requirements dictate that the payload be secured to prevent unplanned movement. Release in these cases is often accomplished with pyrotechnic devices. The latching approach should accommodate both mechanical and pyrotechnic release. In addition, because of the severe vibrations experienced during launch, the latching system must be preloaded to provide suitable performance.

Several groups have addressed the problem of securing payloads for launch and subsequent operation. Goddard Space Flight Center led an effort culminating in the development of the Express Logistics Carrier (ELC) (in early development it was referred to as the express pallet) to provide a standardized mechanical and electrical interface to payloads and experiments on the International Space Station(ISS) ${ }^{\mathrm{iv}, \mathrm{v}}$. Figure. 1 shows the pallet undergoing checkout prior to launch. In addition, the pallet can be used to secure payloads for launch and subsequent transport to the ISS as shown in Fig. 2. Building on these ideas, a system will be introduced which extends the applicability of a latching system to all phases of the payload handling cycle. 


\section{An Example of a Reusable Latch and Decking System}

In this section, a system will be described that embodies the attributes listed previously. In laboratory tests discussed at the end of the paper, first generation hardware has been shown to be robust. This system uses the payload mass to actuate the latches in a gravity environment; however, this does not preclude design of a system which electrically actuates the latches if desired.

The latch and carrier system are designed as an integrated system. The complex portion of the system is affixed to the payload in the form of the latch and its associated release mechanism. The carrier system is made as simple and robust as possible and, in the current embodiment, is a hole pattern in passive deck plates. Note that the latch mechanism is only affixed to the payload, while the carrier (deck plate) system is integrated with the ground transportation fixtures, launch vehicle, planetary transportation vehicles and likely the outpost infrastructure. Thus, any complexity in the carrier system is replicated many times, while latch complexity is localized. In the following, the latch and then a concept for the carrier system are described.

\section{A. Gravity Actuated Latch Overview}

Figure 3 depicts the integrated system components consisting of a latch device and a carrier with interface holes, with the depiction of the carrier shown to illustrate latch operation. The latch body houses the components constituting the latch mechanism and provides a mechanical interface to the payload. In this embodiment of the device the payload interface is provide by a 3-holed tab machined onto the latch body. The latch plunger translates in the latch body and actuates the latch locks through contact of the latch pin with slots in the latch locks. The latch spring is captured in the latch body by the clip ring and forces the latch plunger toward the carrier interface when the release line is unloaded. Details of the operation of these components during typical operations will be discussed in the next section.

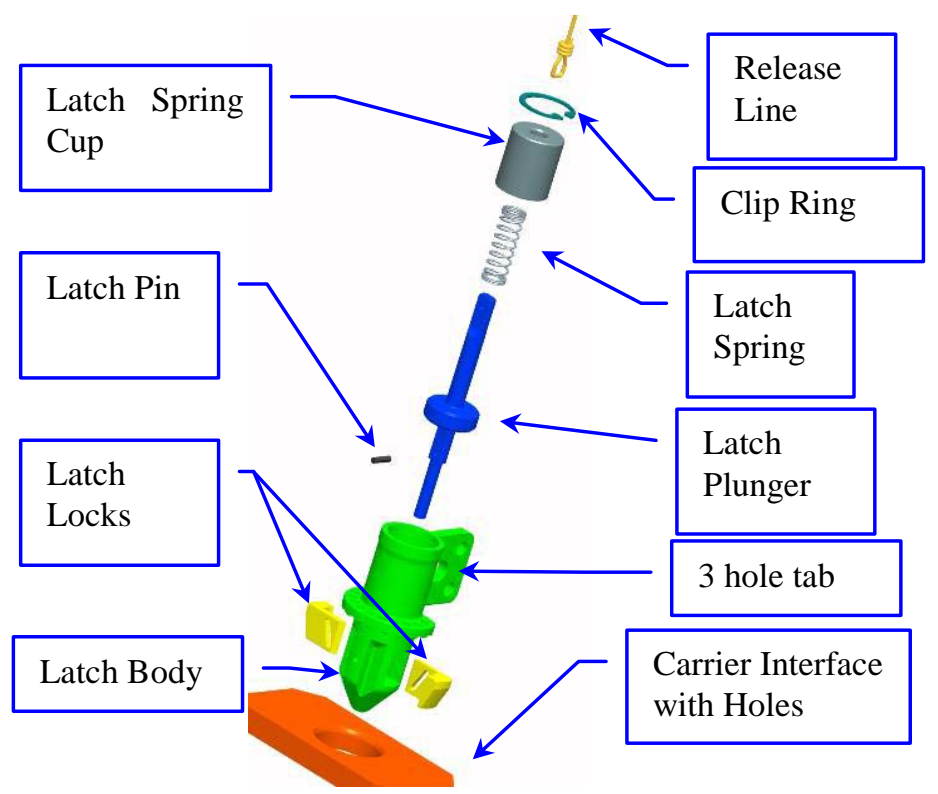

Figure 3. Latch Components

A typical use of the latch unit is shown in Fig. 4, where 3 latches are visible at the base of a payload as it is lowered toward the carrier interface (formed by a pattern of holes designed to accommodate a wide range of payload sizes). Note that some of the holes in the carrier are larger than others. This accommodates different size latches for different payload sizes and masses. It is also straight forward to accommodate different carrier interface thicknesses. In this depiction, the latch is approaching the carrier interface vertically; however, the device has design features to accommodate a variety of approach and entry angles.

In Fig. 4, the release lines are directly connected to a hook on the top of the payload. As the payload is lifted using the lifting hook, the hook initially slides on the center post (visible in the cross sectional view at the upper right) thus pulling on the release lines and releasing the latches. By design, the release lines do not carry the weight of the payload, the lifting hook and lift pins do. This enables the lifting hook to engage the hard-points at the top of the payload used for terrestrial handling. To reduce weight during launch, a heavy duty hook can be used for terrestrial operations and a more lightweight hook integrated prior to launch for planetary operations.

\section{B. Sequence of Latch Operation}

The latch is released or engaged by action of the latch plunger of Fig. 3 in response to either tension on the release line or extension of the latch spring (for engagement). Figure 5 depicts the latch (that would be attached to a payload and has been hidden for clarity) as it approaches the carrier interface. On the right of the figure the latch mechanism is shown in cross section where the latch spring has been compressed by tension on the release line. On the left of the figure, a side view of the latch approaching the carrier interface is shown. When the hook is lifted, the initial hook travel guided by the center post to the top of the lift pin, provides enough tension in the release line to overcome the latch spring, thus retracting the plunger as shown. It may also be desirable to electrically actuate the plunger or release lines to allow engagement. The design does not preclude this alternate realization. 


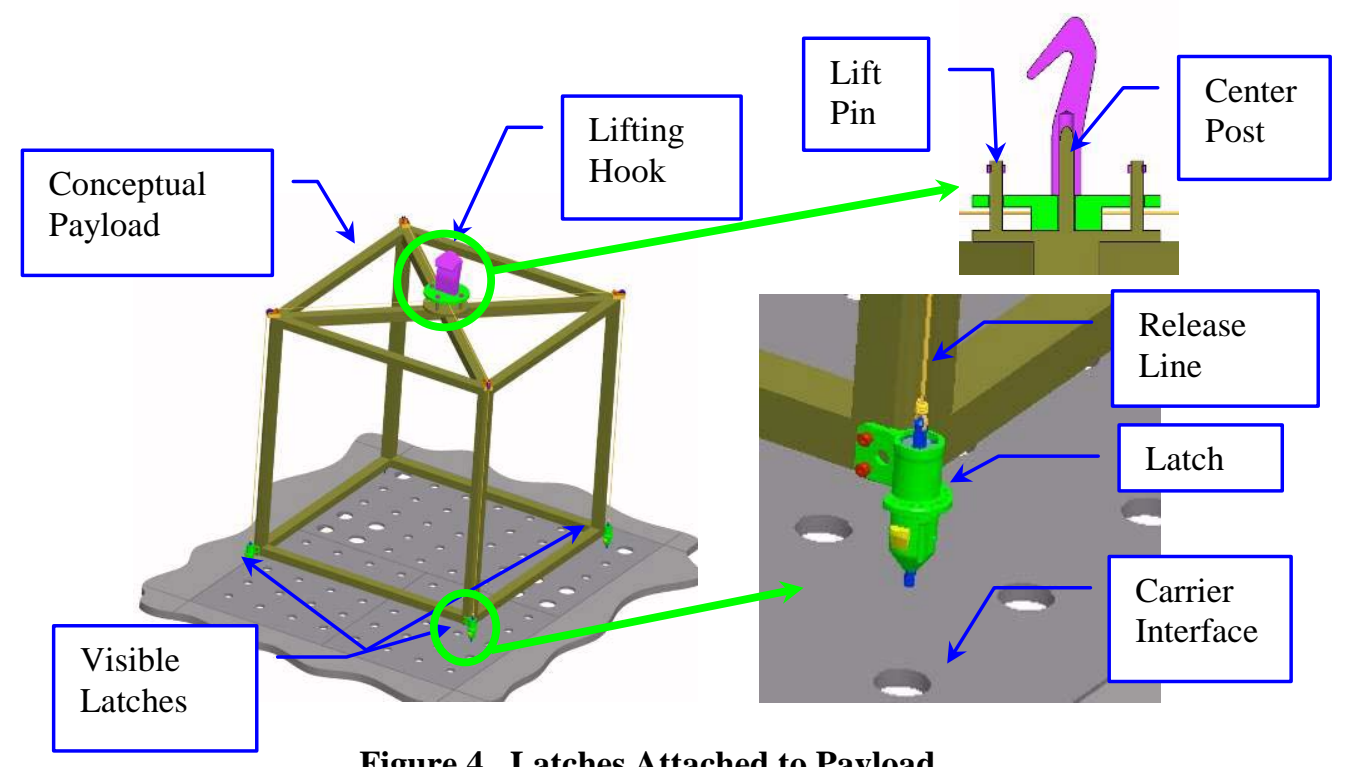

Figure 4. Latches Attached to Payload

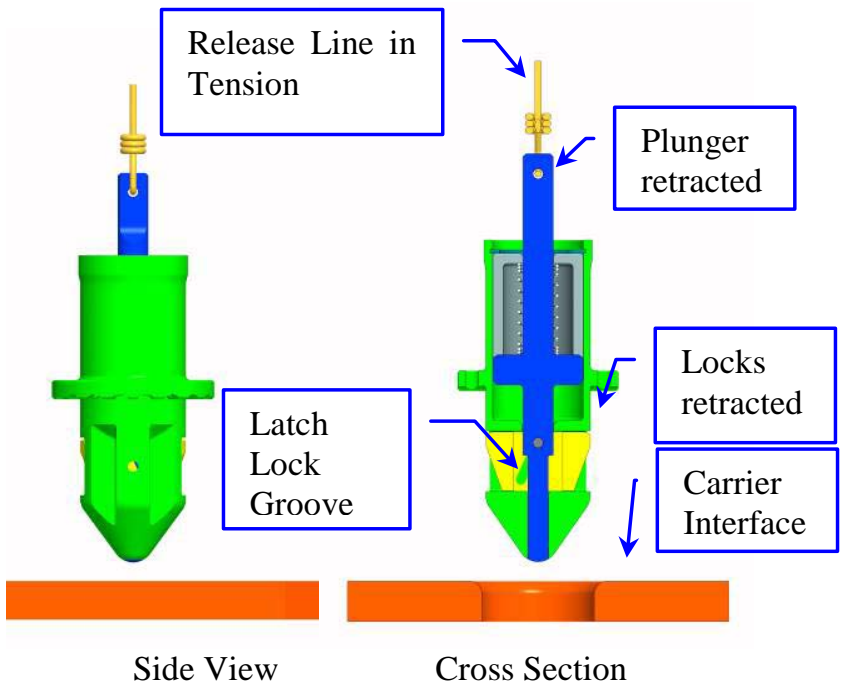

Figure 5. Latch Approaching Carrier Interface

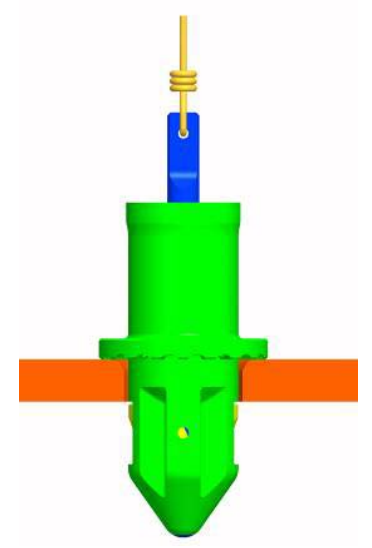

Side View

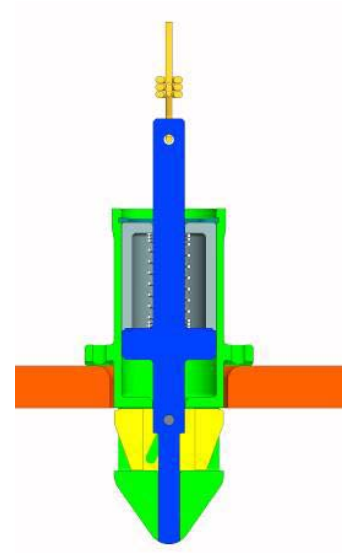

Cross Section

Figure 6. Inserted into Carrier Interface

Figure 6 depicts the latch inserted into the carrier interface just prior to releasing the tension on the release line. As tension is released, the latch spring extends and pushes the latch plunger downward, thus extending the latch locks to secure the latch to the carrier interface as shown in Fig. 7. The latch locks are forced outward by the action of the latch pin sliding in the grooves of the latch locks. The latch pin is affixed to the latch plunger so that as the latch plunger moves up or down, the latch pin rides in the groove of the latch lock, thus retracting or extending the latch locks. The latch is shown in a side view (on the left), cross section (in the center) and an isometric view (on the right) in Fig. 7.

Removing a payload is accomplished by reversing this sequence. As tension is applied to the release line, for example as the payload is lifted using the hook, the latch plunger is pulled upward, retracting the latch locks and freeing the payload from the carrier interface. 


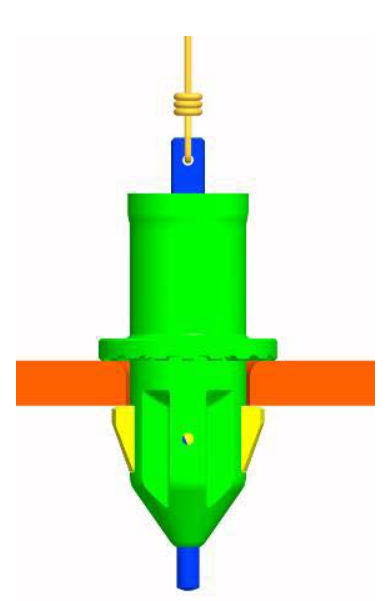

Side View

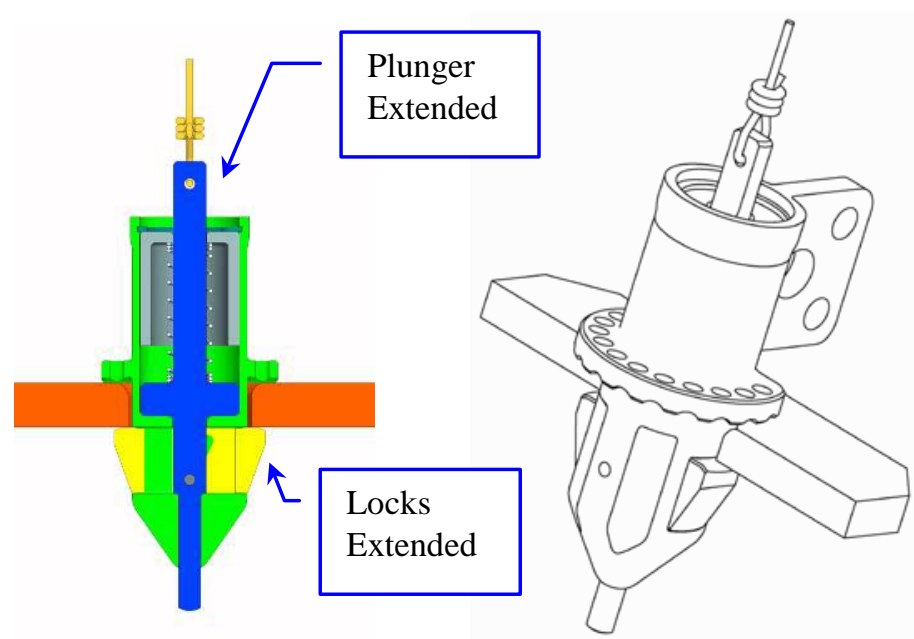

Cross Section

Orthoscopic View

Figure 7. Latch Locked to Carrier Interface

\section{Unique Features of Latch}

The latch has several unique features designed to improve operational robustness and increase the versatility of the device.

First, the latch plunger extends through the tip of the latch. In the locked state, it is possible to strike the plunger and drive it upward to overcome a jam. Alternatively, extension of the latch plunger past the latch body enables the plunger to be directly connected to an explosive release, in effect hard coupling the plunger and thus the payload. This is envisioned to be used during the launch phase to preload the payload to the launch vehicle carrier, potentially by adding threads to the plunger, resulting in the system shown in Fig. 8. The washer shown in the figure can provide vibration attenuation and preload or be used with springs as required.

Second, the latch body and latch plungers are sloped forming a conical shape which enhances passive alignment of the latch during approach. In addition, in situations where the payload is not lifted from the top, which applies tension to the release lines, the latches may be back driven by pressing the latch downward forcing the latch locks to retract. As the locks are back driven, the latch spring is compressed and then once the latch locks pass through the carrier, they move outward as the latch spring extends securing the payload to the carrier interface.

Third, the latch motion is perpendicular to the release direction. Thus, unless released, the latch body will fail before the payload is freed.

Fourth, as shown in the image on the right of Fig. 7, the latch body includes grooves and a flange with scallops and holes to facilitate automatic transport of dirt away from the mating surfaces.

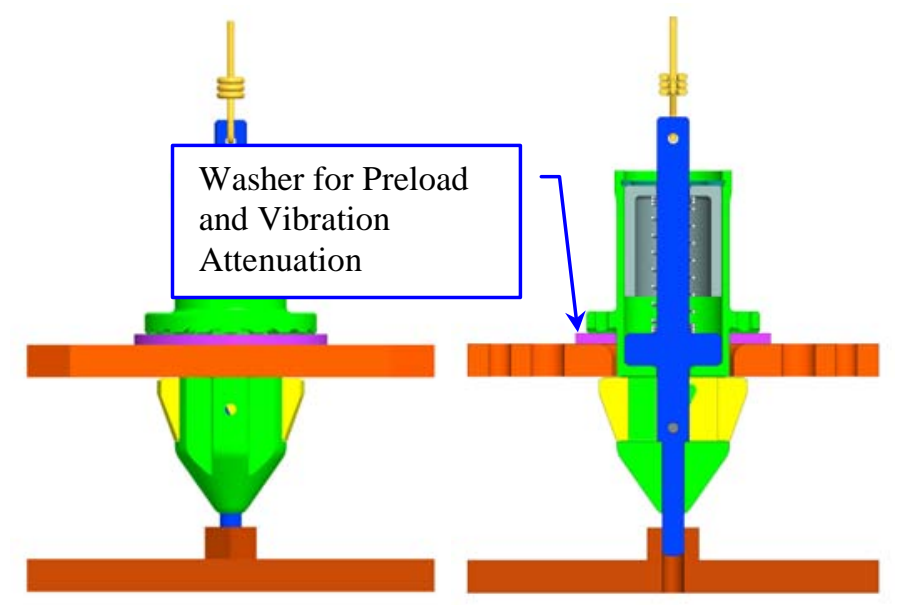

Figure 8. Latch Locked to Carrier Interface

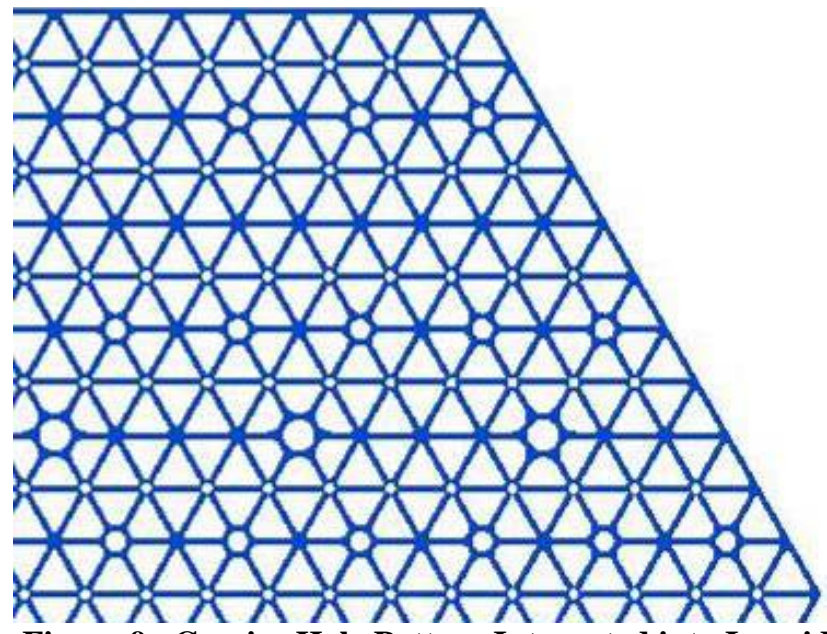

Figure 9. Carrier Hole Pattern Integrated into Isogrid Deck 


\section{Carrier Overview}

The carrier works in synergy with the latch to form a system for securing payloads to a variety of vehicles. Two potential approaches to carrier design are discussed, both compatible with the latch just described.

The first carrier design uses a standardized series of hole patterns over the carrier surface as illustrated in Fig. 9. Note that several sizes of holes are shown, to accommodate different size latches. The payloads accommodate this hole pattern by proper location of the latches on the payload. The latches do not need to be located on the corners of the payload, nor the perimeter. This scheme integrates well with both an isogrid, as shown in the figure or an orthogrid pattern. In general, as the spacing between interface locations increases, the number of latches and their associated mass penalty decreases.

The second carrier design is illustrated in Fig. 10 and uses customized panels that are

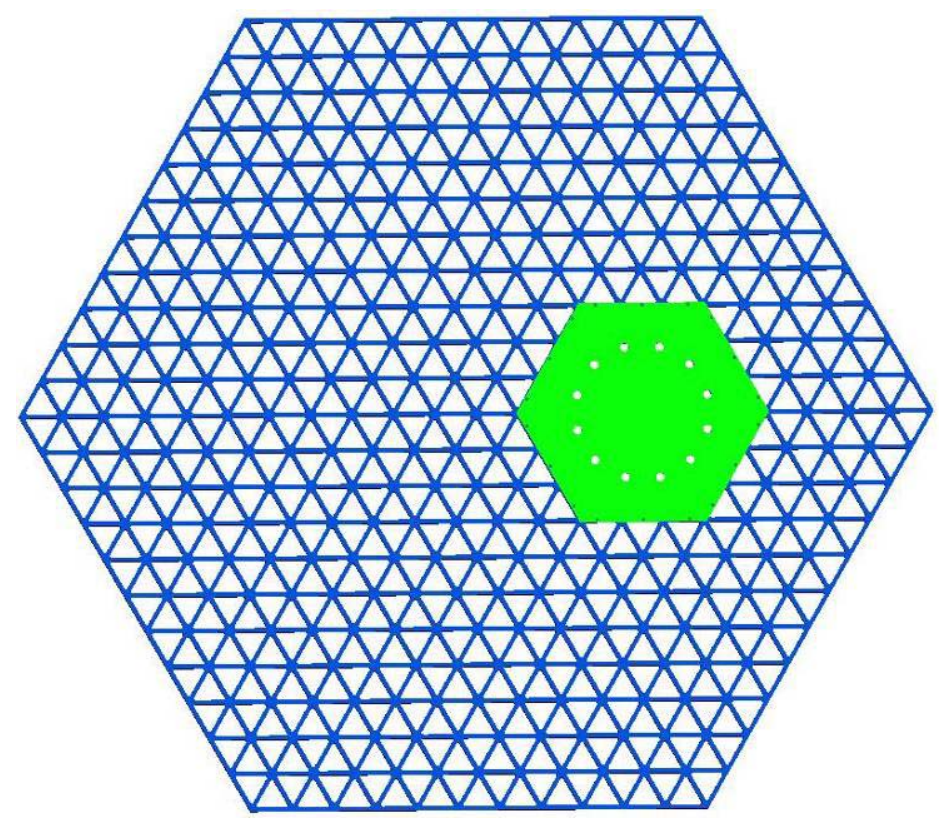

Figure 10. Customized Carrier Panel on Isogrid Deck attached to the underlying support structure (in this case an isogrid). In the figure, a circular pattern is shown which could be used to secure a spherical or cylindrical tank. This approach to carrier design has the potential to improve packaging density at the expense of requiring two designs operations. First, design of a latch to payload interface and second, design of the carrier panels and their interface to the support structure. A more detailed study is required to determine which approach results in the lowest overall mass.

\section{E. Potential Improvements to Latching System}

The hardware that has been described is first generation and exhibits the critical features of a proposed integrated latch and carrier system. As will be shown in the next section the system is operational and fully functional. Subsequent development could improve some aspects of the first generation hardware performance. The size of the hardware could be reduced and alternative materials utilized to reduce the component weight. Expansion into a suite of latches with the ability to provide not only mechanical connectivity, but also electrical and/or fluid connectivity during the latching operation would greatly increase the range of payloads serviced.

\section{Planned tests of the Reusable Latch and Carrier System Hardware}

First Generation hardware is shown in Fig. 11. On the left of the figure the payload is shown, and on the right, details of the latch and release mechanism are shown. Note that the release lines are very thin and not visible in these images.

The weight of the payload is carried by the hook at the top of the payload through the interface to the lifting point

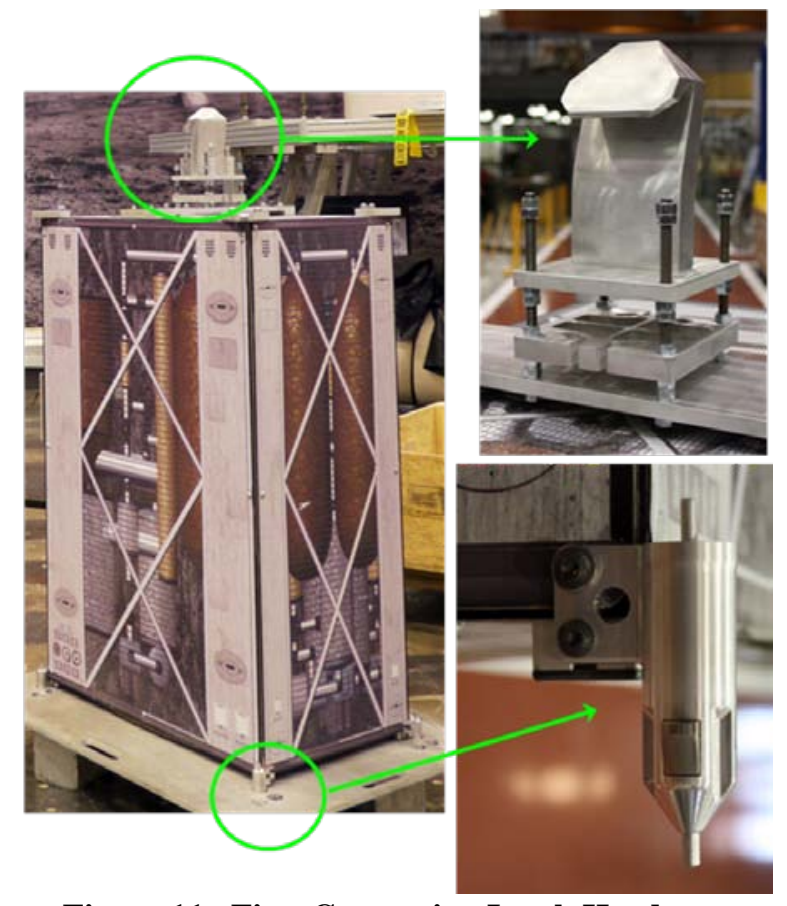

Figure 11. First Generation Latch Hardware Attached to a Payload

at the top. Release lines run from the hook to the latches at the 4 corners of the payload base. The latches in turn, interface with the carrier panel shown in the lower left (which is a plate with holes in this case). 
Initial trials have been performed with a hybrid crane manipulator called the Lunar Surface Manipulation System (LSMS) shown in Fig. 12. vivii $^{\text {Til }}$ The tests have confirmed the robust nature of the connection, which routinely engages and disengages the carrier, both when the carrier is located at ground level as shown and when affixed to an elevated platform. Engagement of the hook and release of the payload occurs at the operating speed of the LSMS, typically taking approximately 15 seconds for engagement, lift and release from the carrier. Tests are planned with the payload located on a sloping carrier to assess the ability of the system to accommodate removal of payloads by an overhead device, such as the LSMS, from uneven terrain with slopes of up to 14 degrees expected to be accommodated.

\section{Summary}

Efficient handling of payloads destined for a planetary surface, such as the moon or mars, requires robust systems to secure the payloads during transport on the ground, in-space and on planetary surfaces. In addition, mechanisms to release the payloads need to be reliable to ensure successful transfer from one vehicle to another. An efficient payload handling strategy must also consider the devices available to support payload handling. Cranes used for overhead lifting are common to all

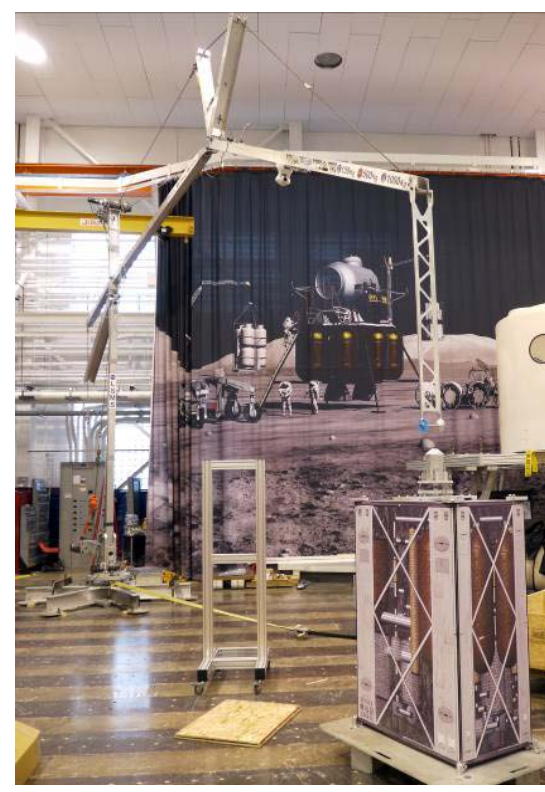

Figure 12. Initial trials with LSMS applications of payload handling on Earth. Similarly, both recent and past studies have demonstrated that devices with comparable functionality will be needed to support lunar outpost operations. A payload handling approach must address all phases of payload management including: ground transportation, launch, planetary transfer and installation in the final system. In addition, storage may be required during any phase of operations. Each of these phases requires the payload to be lifted and secured to a vehicle, transported, released and lifted in preparation for the next transportation or storage phase. A critical component of a successful payload handling approach is a latch and associated carrier system. The latch and carrier system should minimize requirements on the: payload, carrier support structure and payload handling devices; while accommodating a wide range of payload sizes. In addition, the latch should be: small and lightweight, support a method to apply preload, reusable, integrate into a minimal set of hard-points and have manual interfaces to actuate the latch should a problem occur.

A latch and carrier system which meets the requirements just described has been introduced and described in detail. The system supports two approaches to carrier interface design, either a standard hole pattern to accommodate the payloads or use of customized interface panels which reside between the payload and the carrier support structure. A first generation test-bed of a new high performance device that provides the capabilities of a crane and a manipulator, the Lunar Surface Manipulation System (LSMS) has been used to evaluate first generation hardware of the latch and carrier system. All tests have been successful in the early stages of this evaluation. Further tests are planned to evaluate removal of payloads from landers and transporters located on uneven terrain with slopes of up to 14 degrees.

\section{References}

\footnotetext{
i “Moon Crane”, Cranes today magazine Aug. 2008, pp. 23-4.

ii Adinolfi, P.J.; Heinz, F.A. Jr: “Design Study of Special Purpose Systems For the Lunar Surface,” NASA CR-61077, April 30, 1965.

iii Eagle Engineering, “Lunar Surface Construction \& Assembly Equipment Study: Lunar Base Systems Study Task 5.3,” NASA-CR-172105, September, 1988.

iv Gene Cook, "Capabilities and Accommodations on the ISS EXPRESS Pallet”, AIAA-2004-439, 42nd AIAA Aerospace Sciences Meeting and Exhibit, Reno, Nevada, Jan. 5-8, 2004.

${ }^{\vee}$ David Boyle and M. Jacox, “ Express Pallet as an Engineering Test Bed for Spacecraft Technology” AIAA-2004-440 42nd AIAA Aerospace Sciences Meeting and Exhibit, Reno, Nevada, Jan. 5-8, 2004.

${ }^{\text {vi }}$ Doggett, William R.; King, Bruce D.; Jones, Thomas C.; Dorsey, John T.; and Mikulas,Martin M., "Design and Field Test of a Mass Efficient Crane For Lunar Payload Handling and Inspection - The Lunar Surface Manipulation System.” Presented at the AIAA Space 2008 Conference and Exposition, 9 - 11 September 2008, San Diego, California. Available as AIAA- 20087635.

vii Dorsey, John T.; Mikulas, Martin M.; and Doggett, William R., "Preliminary Structural Design Considerations and Mass Efficiencies for Lunar Surface Manipulator Concepts”. Presented at the AIAA Space 2008 Conference and Exposition, 9 - 11 September 2008, San Diego, California. Available as AIAA-2008-7916.
} 